Table 2

Mean and Standard Deviation of Peak Force on Various Contact Surface Diameters (Grams)

\begin{tabular}{rlrrrrr} 
& & \multicolumn{5}{c}{ Diameter $(\mathrm{mm})$} \\
\cline { 3 - 7 } & & \multicolumn{1}{c}{1.6} & \multicolumn{1}{c}{3.2} & 6.4 & 12.7 & 25.4 \\
\hline \multirow{2}{*}{ S1 } & Mean & 153.4 & 154.1 & 154.7 & 155.3 & 156.7 \\
& SD & 13.4 & 13.2 & 12.9 & 12.0 & 12.6 \\
S 2 & Mean & 150.3 & 149.1 & 148.4 & 149.7 & 150.3 \\
& SD & 13.3 & 12.9 & 14.2 & 14.6 & 14.2 \\
\hline
\end{tabular}

pressure sensation, the present procedure clearly generated a considerable range of sensational variation in the emission of force. The invariance in the performance in the face of this variation in pressure sensation provides a dilemma.

Although the present procedure is essentially a free-operant differentiation of response, as Mintz \& Notterman (1965) have noted, the procedure bears similarities to the psychophysical method of average error. The S here "matches" an implicit standard related to sensory feedback from prior responses and the digital feedback of these response magnitudes. The standard deviation then becomes an index of the difference threshold. The proportional difference in pressure sensation between, say, a $150-$ and $160-\mathrm{g}$ (1.47and $1.57-N$ ) response is, of course, constant regardless of the absolute level of pressure. The invariance in performance observed across the variations in absolute pressure could reflect a manifestation of Weber's law. The Ss' resolution of forces is a constant related to constant proportional resolution of pressure. An explanation of this sort is difficult to reconcile with Mintz \& Notterman's (1965) data, where variability was not a constant proportion of the absolute force or, in that case, of absolute pressure as well. Additionally, it is unlikely that a Weber's law sort of invariance would hold true over the range of pressures explored, the highest exceeding the lowest by a factor of 256 .

A possible explanation may rest in the contribution of proprioceptive sensation to force emission. At the $150 \mathrm{~g}$ (1.47-N) level, proprioception may play a predominant role. The invariance of performance may reflect nothing more than the same muscle action in emitting $150 \mathrm{-g}$ responses regardless of the pressure at the fingertip. An implication of this possibility is a range of force emission where pressure variation will determine the precision of the behavior. It is likely that performance at any force level will relate to the acuity of the sensory system most differentially acute or reliable at that level. At low levels of force, for instance, pressure sensation may be the dominant factor in performance, whereas higher force levels may
The ancient art of artificial memory (Yates, 1966) is relevant to modern concern with associative learning in the context of a theoretical evaluation of the concept of mental imagery (Bower, in press; Bugelski, 1970; Paivio, 1969). In this laboratory, the goal of the exploration of the artificial memory system is a search for methods to defeat the effects of neurological disorders that interfere with normal memory (Crovitz, 1970). We have found that self-produced memory maps are not a necessary part of the artificial memory process (Crovitz, 1969), nor are self-produced bizarre images (Briggs, Hawkins, \& Crovitz, 1970). Thus, it is now known that inexperienced $\mathrm{Ss}$ can do quite well in memorizing a long list of words in a single trial using a simplified version of the artificial memory system with memory loci and bizarre mediators supplied by $\mathrm{E}$.

According to Yates (1966), one problem in the use of the artificial memory system remained unsolved in historical times, and led to the greatest difficulties. It is the problem of the capacity of memory loci. While the author of ad Herrenium, the classical source textbook of the artificial memory, wrote that the same set of locations could be used over and over again in memorizing different lists, later mnemonists went to great pains to memorize a great number of loci,

*This paper was delivered at the meetings of the Southern Society for Psychology and Philosophy, Athens, Georgia, April 9, 1971. incorporate proprioceptive acuity. Future research will attempt a further analysis of pressure and proprioceptive sensation in force emission.

\section{REFERENCES}

GELDARD, F. The human senses. New York: Wiley, 1953

MINTZ, D. E., \& NOTTERMAN, J, M. Force differentiation in human subjects. Psychonomic Science, 1965, 2, 289-290. NOTTERMAN, J. M., \& MINTZ, D. E. Dynamics of response. New York: Wiley, 1965 .

\title{
The capacity of memory loci in artificial memory*
}

\author{
HERBERT F. CROVITZ \\ Veterans Administration Hospital, Durham, N.C. 37705
}

The ancient mnemonic system of artificial memory taught a two-part process. First, a set of successive locations was committed to memory; then lists were memorized through making bizarre associations between successive list items and successive locations. This study measures the capacity of locations in artificial memory. A set of 32 English words were the list items. One, 2, 4, 8, 16, or 32 locations were used, as well as a control condition in which no mnemonic instructions were given. The results approximate a normal ogive, with the $50 \%$ point of accurate recall occurring with 8 locations, i.e., four items per location.

Peter of Ravenna in the 15 th century writing that he memorized 100,000 locations when young, and added to them.

Using the peg-word mnemonic, Bower (in press) has shown that up to 20 words may be successfully associated to a single peg. His Ss integrated the words into a story. This was done with each peg exhausted before the next was used. Bower's method must be contrasted sharply with the artificial memory method in which successive locations are associated with successive list words; one by one. If there are $\mathbf{n}$ times as many list words as loci, in the artificial memory system, the set of loci would be used $n$ times, with $n$ runs through the set of loci and successive one-by-one associations made on each run.

The present experiment explores multiple associations to memory loci using the artificial memory system. METHOD

The Ss were 70 Duke University undergraduates who served as part of a course requirement. At the start of their participation, they were entirely naive concerning the mnemonic system of the artificial memory. The Ss were tested individually.

A set of 32 English words was selected as the word list to be memorized in a single trial by all Ss. It was given in a constant order with a period of $10 \mathrm{sec}$ between successive words, using a tape recording. The word list was: apricot, stone, doll, 


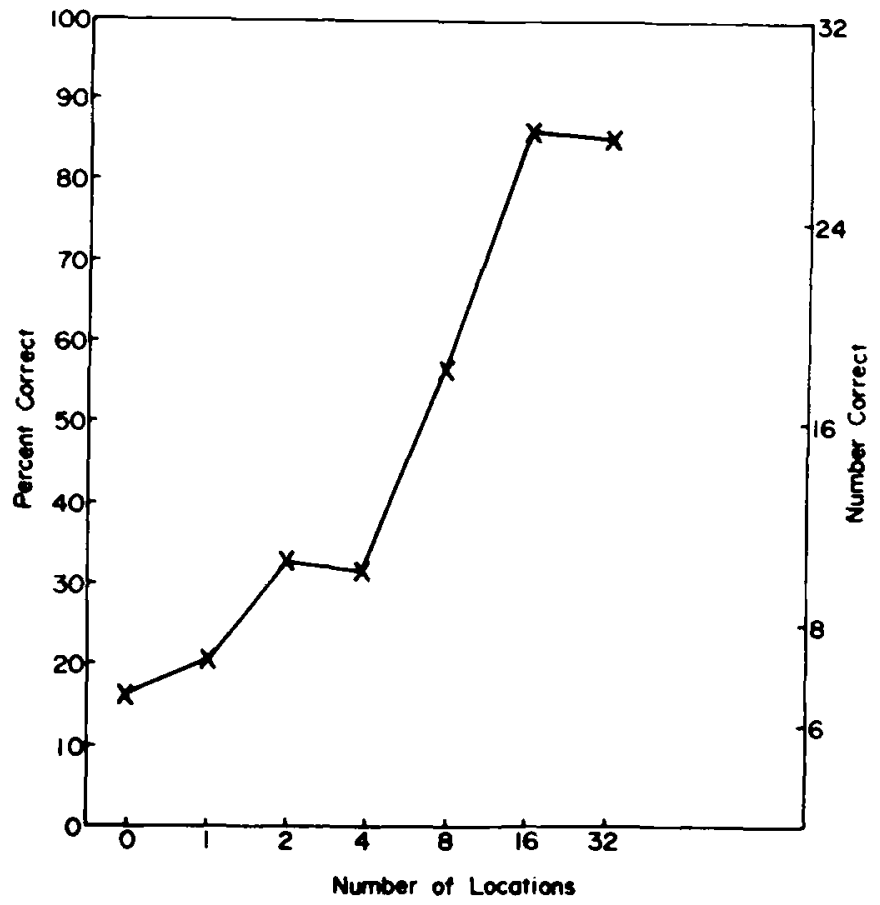

Fig. 1. Recall of list items in their correct place in the list as a function of number of locations.

overcoat, eagle, cabbage, escape, gift, irksome, promise, true, ride, dark, crush, umbrella, sailor, soldier, cedar, mischief, salute, minnow, school, invite, captain, raisin, steep, riot, path, satisfy, peach, sulpher, and perverse.

Another set of 32 items was selected as memory loci, and were typed individually on $3 \times 5 \mathrm{in}$. index cards. They were: PET STORE, PHONE BOOTH, DRESS SHOP, GUN SHOP, HOSPITAL, BOOK SHOP, GAS STATION, NURSERY, DAIRY, CIRCUS TENT, HARDWARE STORE, SIDEWALK CAFE, BUS TERMINAL, DISCOTEQUE, MOTEL, MORTUARY, PERSIAN RUGS, Y.M.C.A., BANK, FLORIST, JAIL, OCULIST, VACANT LOT, BOWLING ALLEY, SODA SHOPPE, NEWSPAPER STAND, ZOO, DRY CLEANER, DIME STORE, FIRE STATION, LOANS, and USED CARS.

Ten Ss were randomly assigned to each of seven groups. The control group was given no mnemonic instructions and no location cards. For the other six groups, $1,2,4,8,16$, or 32 location cards were drawn randomly from the shuffled deck of location cards and placed in a line or lines across the experimental table. The instructions for the mnemonic groups were, "These are your locations. I am going to read you a list of words. I want you to picture them vividly at these successive locations. There will be 32 words, so on the second, or third, etc., time around, depending on the number of locations you chose, you will have to put other items in each location. Try to make bizarre images connecting the locations with the words. Imagine that one of the locations is BATHTUB and the word is whale. You might picture a whale swimming in a bathtub. At the end, you will number a sheet from 1 to 32 and put words down in their correct order. That is, the 17 th word on your paper."

Ss were then asked to explain what they were expected to do, and questions and confusions were treated so that $E$ felt that $S$ understood the directions, but were not elaborated on $E$ then suggested that the location cards be thought of as locations on a street, and suggested that $S$ plan to make mental walks down it in a particular sequence, distributing his "images."

RESULTS AND DISCUSSION

There was a highly significant difference among the conditions with respect to the number of items recalled in their correct position in the list $(F=46.07, \mathrm{df}=6,69, \mathrm{p}<.001)$. However, not all conditions were different from all others. Specifically, there was no significant difference between the control condition (no locations and no mnemonic instructions) and the condition of one location ( 32 words to be associated to the single location). Similarly, there was no significant difference between that I read you will be the 17 th word the conditions of two locations and four locations (16 and 8 items per location, respectively) and between the conditions of 16 and 32 locations ( 2 and 1 item per location, respectively). Between all other possible pairs of conditions, the differences were significant beyond the $1 \%$ level.

Why does performance diminish as more and more "images" are stored in a single location? An analysis of errors strongly suggests that much of the problem resides in the serial ordering of runs through the locations. Even in the control condition, $51 \%$ of list items were retrieved, but to order the list items correctly, given successive runs through a small set of locations, Ss must be able to use the sequence of locations to order the sequence of list items using the artificial memory mnemonic. Less than $20 \%$ of the list items were retrieved and put in their correct place in the list in the control condition. In the conditions of $1,2,4$, and 8 locations, the total number of list words retrieved, ignoring correct sequence, did not significantly change from the control condition, though correct placement of list items did improve, suggesting that the value of the sequential locations resides in the ordering of the items memorized. A significantly higher number of items were recalled, ignoring correct ordering, in the conditions of 16 and 32 locations $(90 \%$ and $81 \%$, respectively), but in these conditions almost all the items recalled were recalled in their correct order.

The tentative conclusion of this study is that a set of locations cannot be used over and over again in artificial memory without introducing sequence errors. However, this conclusion must be taken in the context of an experiment using $S s$ totally inexperienced in the mnemonic system. It remains possible that practice in using the artificial memory mnemonic may increase the ability to preserve serial order.

\section{REFERENCES}

BOWER, G. H. Mental imagery and associative leaming. In Lee Gregg (Ed.), Cognition and learning and memory. New York: Wiley, in press.

BRIGGS, G. G., HAWKINS, S., \& CROVITZ, H. F. Bizarre images in artificial memory. Psychonomic Science, $1970,19,353-354$.

BUGELSKI, B. R. Words and things and images. American Psychologist, 1970, 25, $1002-1006$

CROVITZ, H. F. Memory loci in artificial memory. Psychonomic Science, 1969, 16 , 82-83

CROVITZ, H. F. Memory disorders and the art of memory. Newsletter for Research in Psychology, 1970, 12, 66-69.

PAIVIO, A. Mental imagery in associative learning and memory. Psychological Review, 1969, 76, 241-263.

YATES, F. The art of memory. Chicago: University of Chicago Press, 1966. 